\title{
Modern Lipid Management: A Literature Review
}

\author{
Jahanzeb Malik ${ }^{1}$, Hassan Shabeer ${ }^{1}$, Uzma Ishaq ${ }^{2}$, Humaira Chauhan Sr. ${ }^{3}$, Hina Fatima Akhtar ${ }^{1}$ \\ 1. Cardiology, Rawalpindi Institute of Cardiology, Rawalpindi, PAK 2. Hematology and Medical Oncology, Fauji \\ Foundation Hospital, Rawalpindi, PAK 3. Internal Medicine, KRL Hospital, Islamabad, PAK
}

Corresponding author: Jahanzeb Malik, heartdoc86@gmail.com

\begin{abstract}
Pro-protein convertase subtilisin/Kexin type 9 (PCSK9) inhibitors are relatively new, non-statin, lipidlowering drugs that reduce low-density lipoprotein cholesterol (LDL-C) by 60\%. PCSK9 inhibitors reduce the blood concentrations of cholesterol by the degradation of LDL receptors, which subsequently extracts cholesterol from cells. This leads to cardiovascular risk reduction in various at-risk populations, including atherosclerotic coronary artery disease. Despite their promise for advanced lipid-lowering ability, costeffectiveness is a barrier to their routine use. While searching PubMed, we extracted land-mark trials on two of the anti-PCSK9 monoclonal antibodies, alirocumab and evolocumab. When combined with statins or ezetimibe, they show an exponential fall in LDL-C levels, helping achieve target values in high-risk populations and decreasing cardiovascular adverse events. Ongoing research is exploring the long-term efficacy of these antibodies in established coronary artery disease and familial hypercholesterolemia with more prospects for this novel lipid-lowering therapy.
\end{abstract}

Categories: Cardiology, Allergy/Immunology, Epidemiology/Public Health

Keywords: pcsk9 inhibitors, hyperlipidemia, atherosclerosis, coronary artery disease, hypercholesterolemia, ldl-c, alirocumab, evolocumab

\section{Introduction And Background}

For decades, statins have been the mainstay of treatment for hypercholesterolemia. They decrease cholesterol synthesis through the inhibition of 3-hydroxy-3-methylglutaryl-CoA (HMG-CoA) reductase, a rate-controlling enzyme in the mevalonate pathway [1]. Although they provide a successful reduction in the risk of major cardiovascular events and mortality for the at-risk population, there are patients for whom statins alone or in combination with multiple lipid-lowering agents are inadequate to reduce cardiovascular risk. Some patients have an intolerance to statins due to excessive symptoms and/or liver disease [2]. In those patients, other lipid-lowering agents like bile acid sequestrants or ezetimibe is normally insufficient [3].

Received 07/13/2020 Review began 07/14/2020 Review ended 07/14/2020 Published 07/24/2020

() Copyright 2020 Malik et al. This is an open access article distributed under the terms of the Creative Commons Attribution License CC-BY 4.0., which permits unrestricted use, distribution, and reproduction in any medium, provided the original author and source are credited.
In general cardiology practice, a large number of patients who are at high cardiovascular risk are below the target range for LCL-C levels. Recommended LDL-C should be below $70 \mathrm{mg} / \mathrm{dl}(<1.8 \mathrm{mmol} / \mathrm{L})$ [4] and inadequate control of LDL-C shows a need for more effective treatments. It may also indicate noncompliance to treatment, especially in patients with multiple comorbidities who are taking a lot of medications, apart from the fact that $10 \%$ of the population is also intolerant to statins [5].

High levels of LDL-C are identified as genetic polymorphisms rather than lifestyle choices [6]. In the early 2000s, a genetic sequence was identified, which binds to LDL receptors, thus inhibiting the binding of LDL-C and its removal from the body. This, therefore, raises the concentration of LDL-C in the blood. This protein is named pro-protein convertase subtilisin/Kexin type 9 (PCSK9) [7]. The monoclonal antibodies that target PCSK9 for lowering plasma LDL-C concentration were approved in the European Society of Cardiology in their latest lipid guidelines [8]. Alirocumab and evolocumab are the two anti-PCSK9 monoclonal antibodies recently approved by the US Food and Drug Administration (FDA) for the treatment of high cardiovascular risk patients who do not adequately respond to basic lipid-lowering therapy [9-10]. Evolocumab is also approved for homozygous familial hypercholesterolemia and primary hypercholesterolemia [11].

Both alirocumab and evolocumab, in several landmark trials, have shown marked LDL-C reductions over 10 weeks (up to $70 \%$ ). But this result comes at a high price tag. The cost is estimated to be $\$ 14000$ per patient per year [12]. With only modest reductions in non-fatal atherosclerotic events, there has been strict allocation and regulations to restrict access to these therapies.

This literature review aimed to assess the data available, evaluating the efficacy and safety of anti-PCSK9 monoclonal antibodies in patients with increased LDL-C levels.

\section{Review}

\section{Methods}


For this literature review, randomized control trials were included, and to identify relevant studies, PubMed (2005 to 2020) was searched using “anti-PCSK9 antibodies”, “alirocumab”, and "evolocumab” as search terms. ClinicalTrials.gov was also searched for randomized control trials (RCTs).

For inclusion, all phase 3 trials that showed clear positive primary and secondary outcomes were included. Animal studies were excluded, as were case reports, opinions, editorials, and commentaries. Data on patient demographics and the extent of the disease, outcomes of the drug taken by the patients, and the safety of the patients were studied.

\section{Results}

Seven articles were included in this review (Figure 1). The strength of evidence was found to be good. Several studies evaluated a PCSK9 inhibitor in varying cardiovascular risk patients, who failed to achieve target LDL-C levels with primary lipid-lowering strategies (LDL-C less than $70 \mathrm{mg} / \mathrm{dL}$ ).

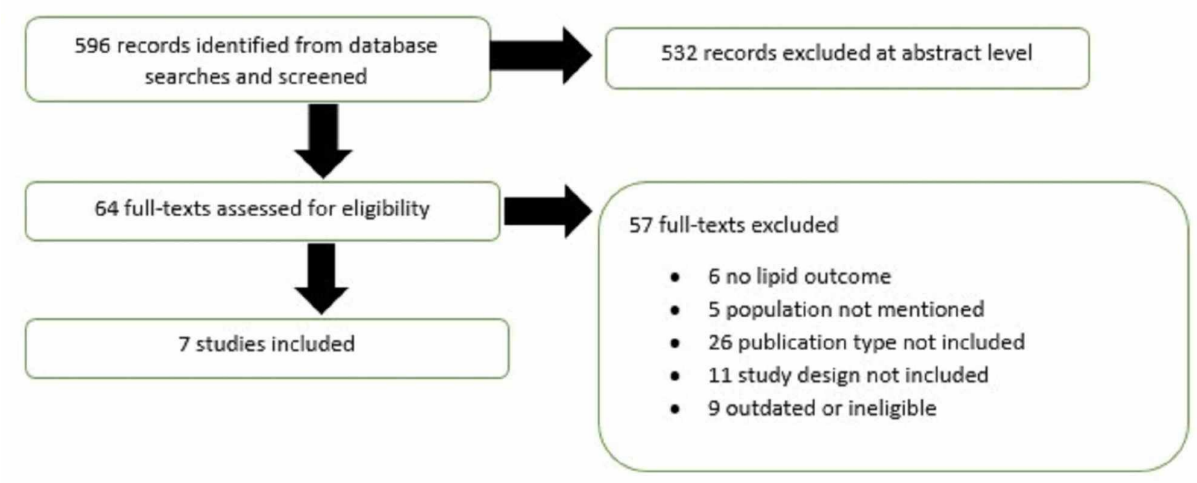

FIGURE 1: Results of the literature search

In patients at high risk for recurrent cardiovascular events, FOURIER (Further Cardiovascular Outcomes Research With PCSK9 Inhibition in Subjects With Elevated Risk; 2017) randomized 27,564 patients aged 40 to 85 years with clinically evident atherosclerotic cardiovascular disease [13-14]. This trial demonstrated that, relative to placebo, evolocumab significantly reduced LDL-C by $59 \%$ at 48 weeks $(\mathrm{P}=0.001)$. Relative to placebo, evolocumab reduced the risk of primary endpoint (1344 patients (9.8\%) vs. 1563 patients (11.3\%); hazard ratio, 0.85 ; $95 \%$ confidence interval $(\mathrm{CI}), 0.79$ to $0.92 ; \mathrm{P}<0.001)$ and the key secondary endpoint $(816$ (5.9\%) vs. 1013 (7.4\%); hazard ratio, 0.80 ; $95 \%$ CI, 0.73 to 0.88 ; $\mathrm{P}<0.001$ ). There was no significant difference between the study groups concerning adverse events.

In another trial (ODYSSEY LONG TERM (Long-term Safety and Tolerability of Alirocumab in High Cardiovascular Risk Patients with Hypercholesterolemia Not Adequately Controlled with Their Lipid Modifying Therapy), 2015), in patients at high risk for cardiovascular events under the diagnosis of heterozygous familial hypercholesterolemia or established coronary artery disease, alirocumab reduced LDL-C levels by $62 \%$ in addition to high-intensity statin therapy with no significant adverse profile $(\mathrm{P}=0.001)[15]$. It also lowered major adverse cardiovascular events as compared to placebo (1.7\% vs. $3.3 \%$; hazard ratio, 0.52 ; $95 \%$ confidence interval, 0.31 to 0.90 ; nominal $\mathrm{P}=0.02$ ). In ODYSSEY COMBO II (Efficacy and Safety of Alirocumab Vs Ezetimibe on Top of Statins in High Cardiovascular Risk Patients with Hypercholesterolemia, 2015), alirocumab was used as an add-on therapy compared with ezetimibe in highrisk patients with inadequately controlled LDL-C levels [16]. At week 24, a 50\% reduction in LDL-C was observed for alirocumab vs. $20 \%$ with ezetimibe. Alirocumab was well-tolerated, with no excess treatmentemergent adverse events.

In two open-label studies (OSLER (Open-Label Study of Long Term Evaluation Against LDL-C Trial) 1 and 2 , 2019), evolocumab reduced LDL-C targets by $61 \%$ more than the standard care at 12 weeks [17-18]. In patients with high cardiovascular risk, the LAPLACE-2 (LDL-C Assessment with PCSK9 Monoclonal Antibody Inhibition Combined With Statin Therapy) study demonstrated that evolocumab, when added to atorvastatin, resulted in high rates of LDL-C targets at 12 weeks [19]. In statin-intolerant patients, two trials (GAUSS-1 and GAUSS-2 (Goal Achievement After Utilizing an Anti-PCSK9 Antibody in Statin Intolerant Subjects)) demonstrated that evolocumab led to higher reductions in LDL-C levels than placebo [20-21].

\section{Discussion}

Studies that were included in this literature review show that alirocumab and evolocumab are tolerated well and are effective in lowering LDL-C, which, in turn, lowers cardiovascular risk in not only the coronary artery disease subset but also in hypercholesterolemia or mixed lipidaemias. Both alirocumab and 


\section{Cureus}

evolocumab reduced LDL-C targets in broad cardiovascular risk levels and comorbidities like diabetes, stroke, and chronic kidney disease.

Some studies have demonstrated a consistent lipid-lowering effect, irrespective of baseline LDL-C levels. For goal achievement in statin-intolerant patients, ODYSSEY ALTERNATIVE (Study of Alirocumab in Patients with Primary Hypercholesterolemia and Moderate, High, or Very High Cardiovascular Risk Who Are Intolerant to Statins) and GAUSS-2 failed to show promising lipid-lowering effect, but the data are still promising given the high baseline levels of LDL-C concentrations [21-22].

Rates of adverse events and of cardiovascular adverse events for both alirocumab and evolocumab were similar to those in the respective control arms of the studies. However, adverse events were lower, with alirocumab monotherapy as compared to a combination with statins. Although rare, treatment-emergent anti-bodies were reported in some studies [23-26]. It was reported at 12\% in the ODYSSEY MONO study [27]. In evolocumab studies, treatment-emergent antibodies were reported in just one patient [28]. In familial hypercholesterolemia patients, the SPIRE (Studies of PCSK9 Inhibition and the Reduction of Vascular Events) trial demonstrated cardiovascular risk reduction with bococizumab [29].

As LDL-C is a recognized risk factor for cardiovascular disease, there is a significant reduction in cardiovascular events, but putting the study limitations into context, results should be interpreted cautiously [30]. Different methodologies were used in different trials and cardiovascular risk parameters were variably defined. To overcome this difference, analyzing the data according to the patient group is a good strategy. However, patient demographics also varied in different trials.

Study details and results are summarized in Table 1.

\begin{tabular}{|c|c|c|c|}
\hline Trial Name & $\begin{array}{l}\text { Sample Size (n), End } \\
\text { Point }\end{array}$ & PCSK9 Inhibitor Dose & $\begin{array}{l}\text { Difference in LDL-C } \\
\text { Change }\end{array}$ \\
\hline FOURIER [13] & $\mathrm{N}=27,56448$ weeks & $\begin{array}{l}\text { Evolocumab } 140 \mathrm{mg} \text { once every two weeks or } 420 \mathrm{mg} \text { once } \\
\text { every one month }\end{array}$ & $-59 \%, p=0.001$ \\
\hline $\begin{array}{l}\text { ODYSSEY LONG } \\
\text { TERM [15] }\end{array}$ & $\mathrm{N}=812,78$ weeks & Alirocumab $150 \mathrm{mg}$ once every two weeks & $-62.6 \%, p=0.0842$ \\
\hline $\begin{array}{l}\text { ODYSSEY COMBO II } \\
{[16]}\end{array}$ & $\mathrm{N}=720,52$ weeks & Alirocumab $75 \mathrm{mg}$ once every two weeks & $-50.6 \%, p=0.0001$ \\
\hline OSLER [18] & $\mathrm{N}=1,255,1$ years & Evolocumab $420 \mathrm{mg}$ once every one month & $-56 \%, p=0.001$ \\
\hline LAPLACE-2 [19] & $\mathrm{N}=2067,12$ week & Evolocumab $140 \mathrm{mg}$ Q2W or $420 \mathrm{mg}$ once every one month & -66 to $75 \%, p=0.001$ \\
\hline GAUSS-1 [20, 21] & $\mathrm{N}=160,12$ week & Evolocumab $280 \mathrm{mg}, 350 \mathrm{mg}, 420 \mathrm{mg}$ once every one month & $-67 \%, p=0.001$ \\
\hline c-1 [29] & $\mathrm{N}=17,000$ & Bococizumab $150 \mathrm{mg}$ once every two weeks & $-43 \% p=0.87$ \\
\hline $\begin{array}{l}\text { LDL-C: Low-Density Lipo } \\
\text { Elevated Risk; ODYSSEY } \\
\text { Hypercholesterolemia No } \\
\text { Ezetimibe on Top of Stati } \\
\text { Against LDL-C Trial; LAPL } \\
\text { Achievement After Utilizin } \\
\text { Events }\end{array}$ & $\begin{array}{l}\text { protein Cholesterol; FOURI } \\
\text { LONG TERM: Long-term S } \\
\text { t Adequately Controlled wi } \\
\text { ns in High Cardiovascular F } \\
\text {-ACE: LDL-C Assessment v } \\
\text { g an Anti-PCSK9 Antibody }\end{array}$ & $\begin{array}{l}\text { IER: Further Cardiovascular Outcomes Research With PCSK9 Inhibiti } \\
\text { aafety and Tolerability of Alirocumab in High Cardiovascular Risk Pati } \\
\text { th Their Lipid Modifying Therapy; ODYSSEY COMBO II: Efficacy and } \\
\text { Risk Patients with Hypercholesterolemia; OSLER: Open-Label Study } \\
\text { with PCSK9 Monoclonal Antibody Inhibition Combined With Statin Th } \\
\text { in Statin Intolerant Subjects; SPIRE: Studies of PCSK9 Inhibition an }\end{array}$ & $\begin{array}{l}\text { Ion in Subjects With } \\
\text { ents With } \\
\text { Safety of Alirocumab Vs. } \\
\text { of Long-Term Evaluation } \\
\text { erapy; GAUSS: Goal } \\
\text { d the Reduction of Vascular }\end{array}$ \\
\hline
\end{tabular}

\section{Conclusions}

This literature review provides an overview of all landmark trials for alirocumab and evolocumab, two antiPCSK9 monoclonal antibodies approved by the FDA. Two large studies, named PROFICIO (Program to Reduce LDL-C and Cardiovascular Outcomes Following Inhibition of PCSK9 In Different Populations) and ODYSSEY, are further investigating these drugs. Using anti-PPCSK9 antibodies as an add-on therapy to statins or ezetimibe, it will allow more patients to achieve their LDL-C goal, with fewer adverse effects. In the statin-intolerant population, their drugs used as monotherapy can significantly reduce target LDL-C levels, conferring reduced cardiovascular risk.

\section{Additional Information}




\section{Disclosures}

Conflicts of interest: In compliance with the ICMJE uniform disclosure form, all authors declare the following: Payment/services info: All authors have declared that no financial support was received from any organization for the submitted work. Financial relationships: All authors have declared that they have no financial relationships at present or within the previous three years with any organizations that might have an interest in the submitted work. Other relationships: All authors have declared that there are no other relationships or activities that could appear to have influenced the submitted work.

\section{Acknowledgements}

We would like to thank Dr. Nismat Javed for her immense contribution to this review.

\section{References}

1. Zhao M, Jiang K, Song W, et al.: Two transcripts of HMG-CoA reductase related with developmental regulation from Scylla paramamosain: evidences from cDNA cloning and expression analysis. IUBMB Life. 2015, 67:954-965. 10.1002/iub.1452

2. Mancini GB, Baker S, Bergeron J, et al.: Diagnosis, prevention, and management of statin adverse effects and intolerance: proceedings of a Canadian Working Group Consensus Conference. Can J Cardiol. 2011, 27:635662. 10.1016/j.cjca.2011.05.007

3. Almeida JT, Esteves AL, Martins F, Palma I: Approach to patients with statin intolerance: evidence-based review. Acta Med Port. 2020, 33:49-57. 10.20344/amp.10376

4. Bianconi V, Banach M, Pirro M, International Lipid Expert Panel (ILEP): Why patients with familial hypercholesterolemia are at high cardiovascular risk? Beyond LDL-C levels. Trends Cardiovasc Med. 2020, [Epub ahead of print]:10.1016/j.tcm.2020.03.004

5. Roh JW, Chun KH, Kang M, et al.: PRavastatin versus FlUVastatin after statin intolerance: the PRUVIntolerance Study with propensity score matching. Am J Med. 2019, 132:1320-1326.

10.1016/j.amjmed.2019.06.003

6. Tachibana-Iimori R, Tabara Y, Kusuhara H, et al.: Effect of genetic polymorphism of OATP-C (SLCO1B1) on lipid-lowering response to HMG-CoA reductase inhibitors. Drug Metab Pharmacokinet. 2004, 19:375-380. 10.2133/dmpk.19.375

7. Duellman SJ, Machleidt T, Cali JJ, Vidugiriene J: Cell-based, bioluminescent assay for monitoring the interaction between PCSK9 and the LDL receptor. J Lipid Res. 2017, 58:1722-1729. 10.1194/jlr.D074658

8. Rallidis LS: The changing landscape of lipid-lowering therapy after the new ESC/EAS guidelines for the management of dyslipidaemias: launching the era of triple hypolipidaemic therapy in very high risk patients. Atherosclerosis. 2020, 292:231-233. 10.1016/j.atherosclerosis.2019.11.009

9. Guedeney P, Sorrentino S, Giustino G, et al.: Indirect comparison of the efficacy and safety of alirocumab and evolocumab: a systematic review and network meta-analysis. Eur Heart J Cardiovasc Pharmacother. 2020, [Epub ahead of print]:24. 10.1093/ehjcvp/pvaa024

10. Jetty V, Glueck CJ, Lee K, et al.: Eligibility for alirocumab or evolocumab treatment in 1090 hypercholesterolemic patients referred to a regional cholesterol treatment center with LDL cholesterol $\geqslant 70$ $\mathrm{mg} / \mathrm{dL}$ despite maximal-tolerated LDL-cholesterol-lowering therapy. Vasc Health Risk Manag. 2017;13, 247 253. 10.2147/VHRM.S133690

11. Gaudet D, Langslet G, Gidding SS, et al.: Efficacy, safety, and tolerability of evolocumab in pediatric patients with heterozygous familial hypercholesterolemia: rationale and design of the HAUSER-RCT study. J Clin Lipidol. 2018, 12:1199-1207. 10.1016/j.jacl.2018.05.007

12. Whayne TF: Outcomes, access, and cost issues involving PCSK9 inhibitors to lower LDL-cholesterol . Drugs. 2018, 78:287-291. 10.1007/s40265-018-0867-9

13. Bergmark BA, O'Donoghue ML, Murphy SA, et al.: An exploratory analysis of proprotein convertase subtilisin/Kexin type 9 inhibition and aortic stenosis in the FOURIER trial. JAMA Cardiol. 2020, 5:1-6. 10.1001/jamacardio.2020.0728

14. Sabatine MS, Giugliano RP, Keech AC, et al.: Evolocumab and clinical outcomes in patients with cardiovascular disease. N Eng J Med. 2017, 376:1713-1722. 10.1056/NEJMoa1615664

15. Taskinen MR, Del Prato S, Bujas-Bobanovic M, et al.: Efficacy and safety of alirocumab in individuals with type 2 diabetes mellitus with or without mixed dyslipidaemia: analysis of the ODYSSEY LONG TERM trial. Atherosclerosis. 2018, 276:124-130. 10.1016/j.atherosclerosis.2018.07.017

16. El Shahawy M, Cannon CP, Blom DJ, et al.: Efficacy and safety of alirocumab versus ezetimibe over 2 years (from ODYSSEY COMBO II). Am J Cardiol. 2017, 120:931-939. 10.1016/j.amjcard.2017.06.023

17. Koren MJ, Sabatine MS, Giugliano RP, et al.: Long-term efficacy and safety of evolocumab in patients with hypercholesterolemia. J Am Coll Cardiol. 2019, 74:2132-2146. 10.1016/j.jacc.2019.08.1024

18. Hirayama A, Yamashita S, Inomata H, et al.: One-year efficacy and safety of evolocumab in Japanese patients - a pooled analysis from the open-label extension OSLER studies. Circ J. 2017, 81:1029-1035. 10.1253/circj.CJ-16-1016

19. Robinson JG, Nedergaard BS, Rogers WJ, et al.: Effect of evolocumab or ezetimibe added to moderate- or high-intensity statin therapy on LDL-C lowering in patients with hypercholesterolemia. The LAPLACE-2 randomized clinical trial. JAMA. 2014, 311:1870-1882. 10.1001/jama.2014.4030

20. Sullivan D, Olsson AG, Scott R, et al.: Effect of a monoclonal antibody to PCSK9 on low-density lipoprotein cholesterol levels in statin-intolerant patients. The GAUSS randomized trial. JAMA. 2012, 308:2497-2506. 10.1001/jama.2012.25790

21. Stroes E, Colquhoun D, Sullivan D, et al.: Anti-PCSK9 antibody effectively lowers cholesterol in patients with statin intolerance: the GAUSS-2 randomized, placebo-controlled phase 3 clinical trial of evolocumab. J Am Coll Cardiol. 2014, 63:2541-2548. 10.1016/j.jacc.2014.03.019

22. Moriarty PM, Thompson PD, Cannon CP, et al.: Efficacy and safety of alirocumab vs ezetimibe in statin- 
intolerant patients, with a statin rechallenge arm: the ODYSSEY ALTERNATIVE randomized trial. J Clin Lipidol. 2015, 9:758-769. 10.1016/j.jacl.2015.08.006

23. Roth EM, Taskinen MR, Ginsberg HN, et al.: Monotherapy with the PCSK9 inhibitor alirocumab versus ezetimibe in patients with hypercholesterolemia: results of a 24 week, double-blind, randomized phase 3 trial. Int J Cardiol. 2014, 176:55-61. 10.1016/j.ijcard.2014.06.049

24. Kastelein JJ, Ginsberg HN, Langslet G, et al.: ODYSSEY FH I and FH II: 78 week results with alirocumab treatment in 735 patients with heterozygous familial hypercholesterolaemia. Eur Heart J. 2015, 36:29963003. 10.1093/eurheartj/ehv370

25. Farnier M, Jones P, Severance R, et al.: Efficacy and safety of adding alirocumab to rosuvastatin versus adding ezetimibe or doubling the rosuvastatin dose in high cardiovascular-risk patients: The ODYSSEY OPTIONS II randomized trial. Atherosclerosis. 2016, 244:138-146. 10.1016/j.atherosclerosis.2015.11.010

26. Bays H, Gaudet D, Weiss R, et al.: Alirocumab as add-on to atorvastatin versus other lipid treatment strategies: ODYSSEY OPTIONS I randomized trial. J Clin Endocrinol Metab. 2015, 100:3140-3148. 10.1210/jc.2015-1520

27. Roth EM, McKenney JM: ODYSSEY MONO: effect of alirocumab $75 \mathrm{mg}$ subcutaneously every 2 weeks as monotherapy versus ezetimibe over 24 weeks. Future Cardiol. 2015, 11:27-37. 10.2217/fca.14.82

28. Hirayama A, Honarpour N, Yoshida M, et al.: Effects of evolocumab (AMG 145), a monoclonal antibody to PCSK9, in hypercholesterolemic, statin-treated Japanese patients at high cardiovascular risk - primary results from the phase 2 YUKAWA study. Circ J. 2014, 78:1073-1082. 10.1253/circj.cj-14-0130

29. Ridker PM, Amarenco P, Brunell R, et al.: Evaluating bococizumab, a monoclonal antibody to PCSK9, on lipid levels and clinical events in broad patient groups with and without prior cardiovascular events: Rationale and design of the Studies of PCSK9 Inhibition and the Reduction of vascular Events (SPIRE) lipid lowering and SPIRE cardiovascular outcomes trials. Am Heart J. 2016, 178:135-144. 10.1016/j.ahj.2016.05.010

30. Abdullah SM, Defina LF, Leonard D, et al.: Long-term association of low-density lipoprotein cholesterol with cardiovascular mortality in individuals at low 10-year risk of atherosclerotic cardiovascular disease. Results from the Cooper Center longitudinal study. Circulation. 2018, 138:2315-2325.

10.1161/CIRCULATIONAHA.118.034273 